\title{
Evaluation of the Methods for Estimating the Fully Reversed Unnotched Fatigue Limits of Steels
}

\author{
Jing Li $\mathbb{D},{ }^{1}$ Yuan-ying Qiu $\mathbb{D},{ }^{1}$ Hai-dong Wang $\mathbb{D},{ }^{2}$ and Zhao-xi Wang $\mathbb{D}^{2}$ \\ ${ }^{1}$ School of Mechatronic Engineering, Xidian University, South Taibai Road, Xi'an 710071, China \\ ${ }^{2}$ Shanghai Spaceflight Precision Machinery Research Institute, No. 76 Guide Road, Shanghai 201600, China \\ Correspondence should be addressed to Jing Li; lijing02010303@163.com
}

Received 10 April 2019; Accepted 17 June 2019; Published 1 July 2019

Academic Editor: Carlos Garcia-Mateo

Copyright (C) 2019 Jing Li et al. This is an open access article distributed under the Creative Commons Attribution License, which permits unrestricted use, distribution, and reproduction in any medium, provided the original work is properly cited.

Experimental determination of the fatigue limit is expensive considering the time and effort involved. To overcome this drawback, several empirical relations based on monotonic tensile properties and/or hardness have been developed for estimating the fatigue limit. In this study, ten estimation methods are evaluated and compared using the experimental data of 171 steels from literature. The scatter band criterion, standard deviation criterion, and statistical analysis are all used to evaluate and compare the predictability of the considered empirical relations. It is found that the fatigue limit can be well correlated with the Brinell hardness. In the absence of Brinell hardness, the ultimate tensile strength-based direct estimation method may be an alternative to estimate the fatigue limit of steels. It is not recommended to estimate the fatigue limit by using the indirect method on the basis of Basquin's fatigue properties estimated by the monotonic tensile properties even though the fatigue limit of steel is often determined by the experimental Basquin's curve at $10^{6}$ cycles.

\section{Introduction}

Fatigue failures occur due to the application of fluctuating stresses that are much lower than the stress required to cause failure during a single application of stress. It has been estimated that fatigue contributes to approximately $90 \%$ of all service failures due to mechanical causes [1]. Fatigue is a problem that can affect any part or component subjected to cyclic loading. Automobiles on roads, aircraft wings and fuselages, ships at sea, nuclear reactors, jet engines, and land-based turbines are all subject to fatigue failures. However, fatigue phenomena are poorly understood until recently since they occur unexpectedly at relatively low stress levels [2]. As the century progressed and the use of metals expanded with the increasing use of machines, more and more failures of components subjected to repeated loads are recorded. Today, structural fatigue has assumed an even greater importance as a result of the ever-increasing use of highstrength materials and the desire for higher performance from these materials.
The fatigue limit has historically been a prime consideration in long-life fatigue design. For a given material, the fatigue limit has an enormous range, depending on surface finish, specimen or component size, type of loading, temperature, corrosive and other aggressive environments, mean stresses, residual stresses, and stress concentrations [1]. Most long-life S-N fatigue data available in the literature consist of fully reversed uniaxial fatigue strengths or fatigue limits of small, highly polished, unnotched specimens based on $10^{6}$ to $5 \times 10^{8}$ cycles to failure in laboratory air environment. Therefore, determination of the fatigue limit from tests will be not only time-consuming but also expensive. In contrast, mechanics properties determined from monotonic tensile tests or hardness tests are more convenient than the fatigue tests. Over the years, many researchers have attempted to develop correlations among the monotonic tensile data or hardness and fatigue limit of materials [3-5]. Such correlations are desirable, considering the amount of time and effort required to obtain the fatigue limit, as compared to the monotonic tensile properties or hardness. If reliable correlations with reasonable accuracy can be 
established, durability performance predictions and/or optimization analyses can be performed, while substantially reducing time and cost associated with material fatigue testing.

In the present study, ten methods utilized for estimating the fully reversed unnotched fatigue limits of steels from monotonic tensile properties or Brinell hardness are analyzed. The study is based on 171 steels which cover quite a range in variables that might affect fatigue behavior such as cyclic hardening/softening characteristics, tensile strengths covering the range from 331 to $2585 \mathrm{MPa}$, and Brinell hardness from 80 to 660 .

\section{The Estimation Methods}

2.1. The Direct Methods. A common procedure to partially compare materials for their fatigue resistance is to plot the unnotched, fully reversed fatigue limit, $\sigma_{\mathrm{L}}$, obtained under similar ideal laboratory conditions described above, versus the ultimate tensile strength, $\sigma_{\mathrm{u}}$. A traditional idea is that the fatigue limit can be increased by raising the strength of a material, either by the chemical composition of the alloy, or by a heat treatment which increases the hardness. An approximation between the fatigue limit and ultimate tensile strength that is often used for steel is given as follows [1]:

$$
\sigma_{\mathrm{L}}=0.5 \sigma_{\mathrm{u}}
$$

A plot of the fatigue limit vs. ultimate tensile strength is provided in Figure 1. The tested data shown in this figure are taken from AISI Bar Steel Fatigue Database [6] (Table 1), American Society for Metals (ASM) Reference Handbook [7] (Table 2), and SAE J1099 Technical Report [8] (Table 3). It can be seen that there is a good deal of scatter in the data and that predictions based on equation (1) have poor agreement with the data and are nonconservative for most of steels.

To improve the predictions, a modified correlation is given by Roessle and Fatemi [4]:

$$
\sigma_{\mathrm{L}}=0.38 \sigma_{\mathrm{u}} \text {. }
$$

Evaluation of equation (2) by using the tested data is also shown in Figure 1. Relatively good predictions can be obtained.

It should be mentioned that traditionally most fatigue limit data are obtained from rotating bending fatigue tests, where here the values are from axial fatigue tests. In axial fatigue tests, lower strengths are often obtained due to reasons such as higher probability of defects associated with larger stressed volume and bending resulting from specimen misalignment. Therefore, the smaller coefficient obtained by Roessle and Fatemi [4] can correlate the tested data better. In the present paper, equation (2) is referred to as the $\sigma_{\mathrm{u}}$-based direct method.

The fatigue limit can also be estimated from the Brinell hardness, HB. An approximation has been suggested to be used [3], which is given as

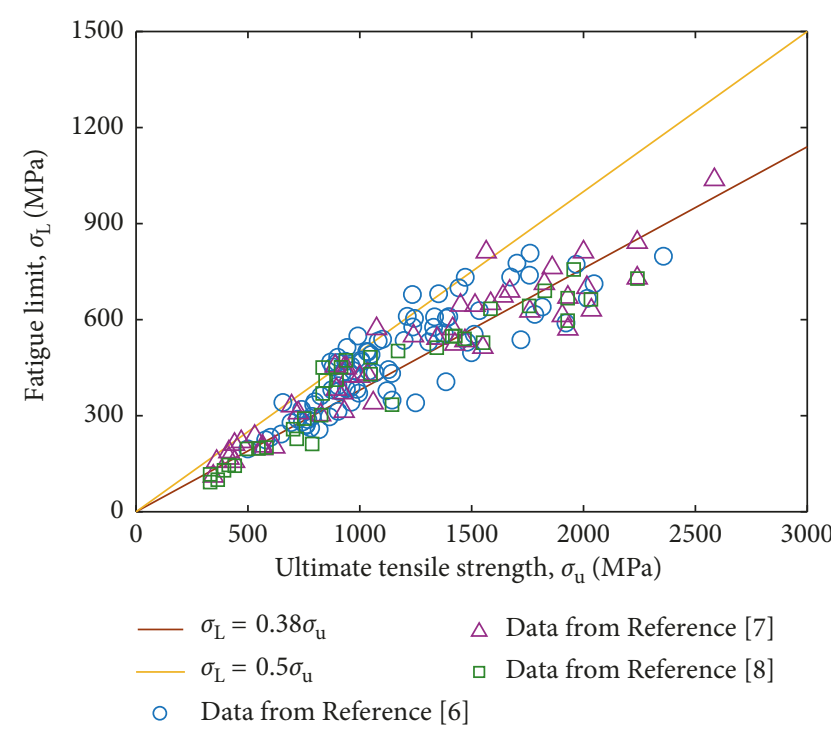

FIgURE 1: Fatigue limit vs. ultimate tensile strength.

$$
\sigma_{\mathrm{L}}=1.72 \mathrm{HB} \text {. }
$$

A plot of the fatigue limit vs. Brinell hardness is provided by using the aforementioned experimental data (Figure 2). Similar to equation (1), there is a poor agreement between the data and the predictions obtained from equation (3). A modification provided by Roessle and Fatemi [4] is given as

$$
\sigma_{\mathrm{L}}=1.43 \mathrm{HB} \text {. }
$$

Evaluations show that equation (4) can provide better predictions (Figure 2). Equation (4) is hereafter referred to as the HB-based direct method.

2.2. The Indirect Methods. In view of the asymptotic character of the S-N curve, the fatigue limit can be defined as the constant stress amplitude at which the fatigue life becomes infinite or fatigue failures do not occur. However, in some cases where fatigue testing must be terminated at a specific large number of cycles, this nonfailure stress amplitude is often referred to as the endurance limit. Even the endurance limit needs not be a fatigue limit, but from the engineering point of view, the term of a fatigue limit is commonly used to refer to either case. Here, the fully unnotched fatigue limit $\sigma_{\mathrm{L}}$ at $10^{6}$ cycles for steels is estimated from the monotonic tensile properties.

Many equations have been proposed to describe the S-N curve. The simplest one is Basquin's equation, which successfully describes the fatigue performance of steels for the high cycle regime [1]:

$$
\sigma_{\mathrm{a}}=\sigma_{\mathrm{f}}^{\prime}\left(2 N_{\mathrm{f}}\right)^{\mathrm{b}}
$$

where $\sigma_{\mathrm{a}}$ is the fatigue stress amplitude, $\sigma_{\mathrm{f}}^{\prime}$ is the fatigue strength coefficient, $b$ is the fatigue strength exponent, and $2 N_{\mathrm{f}}$ is the number of reversals to failure. 
TABLE 1: Monotonic deformation properties and fatigue limit of the selected steels from the AISI Bar Steel Fatigue Database [6].

\begin{tabular}{|c|c|c|c|c|c|c|c|c|}
\hline Materials & $E(\mathrm{GPa})$ & $\sigma_{\mathrm{y}}(\mathrm{MPa})$ & $\sigma_{\mathrm{u}}(\mathrm{MPa})$ & $\sigma_{\mathrm{f}}(\mathrm{MPa})$ & RA (\%) & $\varepsilon_{\mathrm{f}}$ & $\mathrm{HB}$ & $\sigma_{\mathrm{L}}(\mathrm{MPa})$ \\
\hline 1541 & 197 & 471.2 & 783.2 & 1207 & 55.1 & 0.8 & 180 & 260.0 \\
\hline 1541 & 196 & 461 & 905.5 & 1247 & 41.7 & 0.54 & 195 & 311.7 \\
\hline $1050 \mathrm{M}$ & 211 & 449.6 & 821 & 1128 & 49.6 & 0.7 & 238 & 254.5 \\
\hline $1050 \mathrm{M}$ & 202 & 460 & 828.5 & 1065 & 34.1 & 0.416 & 220 & 360.2 \\
\hline $1050 \mathrm{M}$ & 220 & 2100 & 2360 & 2115 & 14.7 & 0.16 & 536 & 796.1 \\
\hline 1090 & 219 & 728.8 & 1090 & 966 & 14 & 0.15 & 259 & 307.1 \\
\hline $1090 \mathrm{M}$ & 217 & 1065 & 1388 & 1427 & 25 & 0.29 & 357 & 404.1 \\
\hline 1090 & 213 & 677.6 & 1147 & 1151 & 21.7 & 0.245 & 309 & 346.8 \\
\hline $1090 \mathrm{M}$ & 212 & 751.3 & 1252.3 & 1169 & 13.6 & 0.146 & 279 & 338.3 \\
\hline $1090 \mathrm{M}$ & 214 & 759.5 & 1124 & 840 & 37.6 & 0.47 & 272 & 376.0 \\
\hline $1141 \mathrm{AL}$ & 216 & 457.4 & 770.6 & 1207 & 57 & 0.85 & 223 & 286.0 \\
\hline $1141 \mathrm{AL}$ & 227 & 814.2 & 924.8 & 1405 & 59 & 0.88 & 277 & 432.0 \\
\hline $1141 \mathrm{Nb}$ & 220 & 417.8 & 695.2 & 1000 & 53 & 0.76 & 199 & 276.6 \\
\hline $1141 \mathrm{Nb}$ & 217 & 602.1 & 801.8 & 1228 & 54 & 0.77 & 241 & 341.8 \\
\hline $1141 \mathrm{~V}$ & 214 & 449.9 & 725.2 & 1087 & 49 & 0.68 & 217 & 287.0 \\
\hline $1141 \mathrm{~V}$ & 215 & 609.7 & 796.8 & 1243 & 58 & 0.88 & 252 & 332.2 \\
\hline $1141 \mathrm{~V}$ & 220 & 493.5 & 789.4 & 1117 & 47 & 0.64 & 229 & 295.8 \\
\hline 1038 & 201 & 330.7 & 582.2 & 898 & 54 & 0.77 & 163 & 222.6 \\
\hline 1038 & 219 & 359.4 & 651.6 & 1051 & 53 & 0.76 & 185 & 240.8 \\
\hline 1038 & 218 & 559.8 & 743 & 1292 & 69 & 1.16 & 245 & 317.6 \\
\hline $10 \mathrm{~V} 45$ & 213 & 465.2 & 764.6 & 1131 & 48 & 0.7 & 212 & 270.4 \\
\hline $10 \mathrm{~V} 45$ & 216 & 605.6 & 908.6 & 1197 & 42 & 0.5 & 269 & 374.3 \\
\hline 1020 & 207 & 294.9 & 502.2 & 849 & 63.5 & 1.01 & 135 & 194.3 \\
\hline $10 \mathrm{~B} 21$ & 212 & 1062.3 & 1104.8 & 1573 & 70.5 & 1.22 & 322 & 536.9 \\
\hline 1022 & 200 & 457.3 & 604.1 & 1587 & 68.7 & 1.16 & 195 & 230.6 \\
\hline 1045 & 209 & 509.6 & 746.6 & 1151 & 62 & 1 & 222 & 278.5 \\
\hline $1151 \mathrm{~V}$ & 206 & 452.3 & 760.8 & 1319 & 50.6 & 0.7 & 205 & 266.9 \\
\hline $15 \mathrm{~V} 24$ & 209 & 645.7 & 877.6 & 1363 & 61.1 & 0.9 & 243 & 379.2 \\
\hline 4130AL & 213 & 1284.5 & 1482.8 & 2055 & 44.3 & 0.6 & 442 & 527.6 \\
\hline 4140 & 207 & 928.9 & 1042.9 & 1519 & 61.3 & 1 & 325 & 437.4 \\
\hline 5140 & 218 & 956.7 & 1039.2 & 1445 & 52.7 & 0.8 & 305 & 496.8 \\
\hline 5150 & 210 & 471.8 & 867.1 & 1382 & 55.8 & 0.8 & 245 & 294.5 \\
\hline $51 \mathrm{~B} 60$ & 200 & 1830 & 1970 & 1968 & 21.6 & 0.23 & 450 & 771 \\
\hline $9254 \mathrm{~V}$ & 206 & 1870 & 2050 & 2118 & 35.1 & 0.4 & 536 & 710.2 \\
\hline 1070 & 207 & 520 & 659 & 1360 & 36.2 & 0.5 & 280 & 339.3 \\
\hline C-70 & 201 & 765 & 964 & 837 & 16.8 & 0.197 & 241 & 340.2 \\
\hline $15 B 35$ & 219 & 866.4 & 939.9 & 1747 & 64.5 & 1.04 & 286 & 467.8 \\
\hline 4620 & 208 & 687.5 & 997.8 & 1530 & 58.2 & 0.872 & 289 & 368.5 \\
\hline 4320 & 202 & 920 & 994 & 1336 & 63 & 0.99 & 188 & 547.1 \\
\hline 4620 & 211 & 892 & 964 & 1651.9 & 61.9 & 0.966 & 289 & 453.5 \\
\hline 5120 & 214 & 780 & 1008 & 1287 & 58 & 0.87 & 252 & 471.8 \\
\hline 9310 & 197 & 804 & 902 & 1418 & 70.8 & 1.23 & 260 & 464.1 \\
\hline 8822 & 204 & 918 & 1131 & 1362 & 56 & 0.88 & 252 & 442.1 \\
\hline 8620 & 203 & 1357 & 1764 & 801.4 & 13.9 & 0.15 & 457 & 805.7 \\
\hline 4320 & 201 & 1344.5 & 1705.3 & 2197.8 & 55.5 & 0.811 & 445 & 774.5 \\
\hline 4140 & 197 & 1297 & 1390 & 1521.5 & 48.3 & 0.66 & 377 & 602.5 \\
\hline 4140 & 200 & 1305 & 1400.5 & 1771 & 48.2 & 0.657 & 380 & 607.5 \\
\hline 4140 & 204 & 1330 & 1537 & 1705.2 & 42.1 & 0.546 & 410 & 626.3 \\
\hline 4140 & 205 & 1314 & 1445.8 & 1754.5 & 52.1 & 0.736 & 390 & 697.3 \\
\hline 4140 & 199 & 1255.1 & 1355.1 & 1332.7 & 38.8 & 0.492 & 370 & 679 \\
\hline 4140 & 201 & 1341 & 1474.2 & 1786.2 & 52.5 & 0.745 & 396 & 730.4 \\
\hline 8620 & 207 & 1401.7 & 1677.2 & 1851.7 & 14.2 & 0.153 & 439 & 731.1 \\
\hline $41 \mathrm{~B} 17 \mathrm{M}$ & 213 & 826.9 & 872.3 & 1304 & 68 & 1.137 & 277 & 465.5 \\
\hline $86 \mathrm{~B} 20$ & 205 & 989 & 1034.1 & 869 & 63.7 & 1.013 & 294 & 498.6 \\
\hline 4140 & 213 & 1669.2 & 1924.3 & 1859 & 15.7 & 0.163 & 477 & 587.7 \\
\hline $41 \mathrm{~B} 17 \mathrm{M}$ & 216 & 863.1 & 904 & 1245.4 & 64.7 & 1.04 & 277 & 480.5 \\
\hline 4140 & 206 & 1290.7 & 1380.4 & 1384.5 & 23.6 & 0.269 & 394 & 554.81 \\
\hline $86 \mathrm{~B} 20$ & 206 & 1198 & 1502 & 968.2 & 59.7 & 0.909 & 401 & 494.8 \\
\hline $5160 \mathrm{H}$ & 209 & 1604.2 & 1761.2 & 1843.8 & 30.2 & 0.359 & 456 & 736.9 \\
\hline 9254 & 206 & 1841 & 2020 & 3644 & 51 & 0.71 & 508 & 664.6 \\
\hline
\end{tabular}


TABle 1: Continued.

\begin{tabular}{|c|c|c|c|c|c|c|c|c|}
\hline Materials & $E(\mathrm{GPa})$ & $\sigma_{\mathrm{y}}(\mathrm{MPa})$ & $\boldsymbol{\sigma}_{\mathrm{u}}(\mathrm{MPa})$ & $\boldsymbol{\sigma}_{\mathrm{f}}(\mathrm{MPa})$ & RA (\%) & $\varepsilon_{\mathrm{f}}$ & $\mathrm{HB}$ & $\sigma_{\mathrm{L}}(\mathrm{MPa})$ \\
\hline $15 \mathrm{~V} 41$ & 193 & 665.5 & 1070.7 & 766.5 & 32.6 & 0.395 & 303 & 431.9 \\
\hline 4140 & 201 & 1363 & 1514 & 2071 & 48 & 0.65 & 405 & 552.4 \\
\hline 1552 & 196 & 420 & 964 & 1526 & 45 & 0.59 & 276 & 387.2 \\
\hline 8620 & 208 & 1566 & 1785 & 2206.4 & 39 & 0.49 & 461 & 614.6 \\
\hline 4140 & 203 & 1157.5 & 1247.3 & 1311.3 & 47.5 & 0.646 & 409 & 601.9 \\
\hline 4140 & 207 & 1167.1 & 1239.5 & 1537 & 47.2 & 0.64 & 417 & 575.2 \\
\hline 4140 & 206 & 1622.2 & 1818.3 & 2016.7 & 42.6 & 0.555 & 477 & 637.83 \\
\hline 4140 & 208 & 1261 & 1332.7 & 1517.6 & 44 & 0.581 & 375 & 574.2 \\
\hline 8822 & 212 & 883.5 & 945.7 & 1170.2 & 67.2 & 1.115 & 297 & 511.2 \\
\hline 8822 & 210 & 990 & 1334.7 & 1542.3 & 46.9 & 0.633 & 301 & 549.3 \\
\hline 8822 & 194 & 1135 & 1215 & 2690 & 59.4 & 0.903 & 338 & 608.5 \\
\hline 8822 & 208 & 1528 & 1723 & 3387 & 50 & 0.672 & 449 & 535.3 \\
\hline 8620 & 212 & 693.5 & 991 & 1411.1 & 54 & 0.776 & 326 & 380.4 \\
\hline 8620 & 210 & 795.8 & 1145.2 & 1585.7 & 50.8 & 0.711 & 352 & 430.2 \\
\hline 8620 & 214 & 989.5 & 1310.8 & 1711.2 & 52 & 0.736 & 382 & 528.2 \\
\hline 4320 & 194 & 620.5 & 905 & 1966 & 59.2 & 0.896 & 261 & 391.8 \\
\hline 4320 & 194 & 745 & 1010 & 1984 & 57 & 0.846 & 288 & 466.3 \\
\hline 4320 & 194 & 867 & 1238 & 2336 & 55.2 & 0.804 & 343 & 676.6 \\
\hline 9310 & 196 & 666 & 883 & 1596 & 61.4 & 0.899 & 255 & 450 \\
\hline 9310 & 196 & 850 & 1085 & 2155 & 57.5 & 0.857 & 306 & 531.5 \\
\hline 9310 & 195 & 990 & 1201 & 2172 & 56.5 & 0.832 & 334 & 533.2 \\
\hline $20 \mathrm{MnCr} 5$ & 194 & 852 & 1053 & 1991 & 56.5 & 0.832 & 299 & 489 \\
\hline $20 \mathrm{MnCr} 5$ & 194 & 1071 & 1337 & 2351 & 52 & 0.74 & 366 & 606.8 \\
\hline 1538MV & 193 & 650.3 & 973.4 & 1038.7 & 28.3 & 0.333 & 285 & 439.1 \\
\hline $1538 \mathrm{MV}$ & 194 & 628 & 943 & 1069.8 & 36.4 & 0.452 & 277 & 382.3 \\
\hline
\end{tabular}

TABle 2: Monotonic deformation properties and fatigue limit of the selected steels from the ASM Handbook [7].

\begin{tabular}{|c|c|c|c|c|c|c|c|}
\hline Materials & $E(\mathrm{GPa})$ & $\sigma_{\mathrm{u}}(\mathrm{MPa})$ & $\sigma_{\mathrm{f}}(\mathrm{MPa})$ & RA (\%) & $\varepsilon_{\mathrm{f}}$ & $\mathrm{HB}$ & $\sigma_{\mathrm{L}}(\mathrm{MPa})$ \\
\hline A538A & 185 & 1515 & 1896 & 67 & 1.1 & 405 & 644.5 \\
\hline A538B & 185 & 1860 & 2137 & 56 & 0.82 & 460 & 762.1 \\
\hline A538C & 180 & 2000 & 2241 & 55 & 0.81 & 480 & 811.3 \\
\hline AM-350 & 180 & 1905 & 2179 & 20 & 0.23 & 496 & 612.4 \\
\hline $\mathrm{H}-11$ & 205 & 2585 & 3172 & 33 & 0.4 & 660 & 1037.2 \\
\hline RQC-100 & 205 & 940 & 1069 & 43 & 0.56 & 290 & 449.1 \\
\hline RQC-100 & 205 & 930 & 1331 & 67 & 1.02 & 290 & 449.1 \\
\hline 10B62 & 195 & 1640 & 1779 & 38 & 0.89 & 430 & 673.4 \\
\hline 1005-1009 & 205 & 360 & 717 & 73 & 1.3 & 90 & 157.2 \\
\hline $1005-1009$ & 205 & 470 & 745 & 66 & 1.09 & 125 & 218.8 \\
\hline 1005-1009 & 200 & 415 & 641 & 64 & 1.02 & 125 & 187.2 \\
\hline $1005-1009$ & 200 & 345 & 848 & 80 & 1.6 & 90 & 111.1 \\
\hline 1015 & 205 & 415 & 724 & 68 & 1.14 & 80 & 167.2 \\
\hline 1020 & 205 & 440 & 710 & 62 & 0.96 & 108 & 156.9 \\
\hline 1040 & 200 & 620 & 1048 & 60 & 0.93 & 225 & 202.0 \\
\hline 1045 & 200 & 725 & 1227 & 65 & 1.04 & 225 & 308.7 \\
\hline 1045 & 200 & 1450 & 1862 & 51 & 0.72 & 410 & 645.0 \\
\hline 1045 & 205 & 1345 & 1862 & 59 & 0.89 & 390 & 541.7 \\
\hline 1045 & 205 & 1585 & 2103 & 55 & 0.81 & 450 & 650.1 \\
\hline 1045 & 205 & 1825 & 2275 & 51 & 0.71 & 500 & 712.7 \\
\hline 1045 & 205 & 2240 & 2723 & 41 & 0.52 & 595 & 841.4 \\
\hline 1144 & 195 & 930 & 1158 & 33 & 0.51 & 265 & 313.3 \\
\hline 1144 & 200 & 1035 & 1517 & 25 & 0.29 & 305 & 429.5 \\
\hline $1541 \mathrm{~F}$ & 205 & 950 & 1276 & 49 & 0.68 & 290 & 423.3 \\
\hline $1541 \mathrm{~F}$ & 205 & 890 & 1276 & 60 & 0.93 & 260 & 455.1 \\
\hline 4130 & 220 & 895 & 1420 & 67 & 1.12 & 258 & 382.4 \\
\hline 4130 & 200 & 1425 & 1820 & 55 & 0.79 & 365 & 523.3 \\
\hline 4140 & 200 & 1075 & 1524 & 60 & 0.69 & 310 & 571.7 \\
\hline 4142 & 200 & 1060 & 1117 & 29 & 0.35 & 310 & 339.8 \\
\hline 4142 & 205 & 1415 & 1827 & 48 & 0.66 & 380 & 571.7 \\
\hline
\end{tabular}


TABle 2: Continued.

\begin{tabular}{|c|c|c|c|c|c|c|c|}
\hline Materials & $E(\mathrm{GPa})$ & $\boldsymbol{\sigma}_{\mathrm{u}}(\mathrm{MPa})$ & $\sigma_{\mathrm{f}}(\mathrm{MPa})$ & RA (\%) & $\varepsilon_{\mathrm{f}}$ & $\mathrm{HB}$ & $\sigma_{\mathrm{L}}(\mathrm{MPa})$ \\
\hline 4142 & 200 & 1550 & 1896 & 47 & 0.63 & 400 & 513.5 \\
\hline 4142 & 200 & 1760 & 1999 & 42 & 0.54 & 450 & 626.5 \\
\hline 4142 & 200 & 2035 & 2068 & 20 & 0.22 & 475 & 629.9 \\
\hline 4142 & 200 & 1930 & 2103 & 37 & 0.46 & 450 & 570.4 \\
\hline 4142 & 205 & 1930 & 2172 & 35 & 0.43 & 475 & 670.0 \\
\hline 4142 & 205 & 2240 & 2654 & 27 & 0.31 & 560 & 729.9 \\
\hline 4340 & 195 & 825 & 1089 & 43 & 0.57 & 243 & 302.4 \\
\hline 4340 & 200 & 1470 & 1558 & 38 & 0.48 & 409 & 534.1 \\
\hline 4340 & 195 & 1240 & 1655 & 57 & 0.64 & 350 & 549.4 \\
\hline 5150 & 195 & 1670 & 1931 & 42 & 0.87 & 430 & 688.9 \\
\hline 52100 & 205 & 2015 & 2193 & 11 & 0.12 & 518 & 700.4 \\
\hline 9262 & 205 & 925 & 1041 & 14 & 0.16 & 260 & 371.2 \\
\hline 9262 & 195 & 1000 & 1220 & 33 & 0.41 & 280 & 423.0 \\
\hline 9262 & 200 & 1565 & 1855 & 32 & 0.38 & 410 & 811.3 \\
\hline $950 \mathrm{C}$ & 205 & 565 & 931 & 64 & 1.03 & 159 & 205.1 \\
\hline $950 \mathrm{C}$ & 205 & 565 & 1000 & 69 & 1.19 & 150 & 196.6 \\
\hline $950 \mathrm{X}$ & 205 & 440 & 752 & 65 & 1.06 & 150 & 210.5 \\
\hline $950 \mathrm{X}$ & 205 & 530 & 1000 & 72 & 1.24 & 156 & 235.5 \\
\hline $980 \mathrm{X}$ & 195 & 695 & 1220 & 68 & 1.15 & 225 & 330.5 \\
\hline
\end{tabular}

TABle 3: Monotonic deformation properties and fatigue limit of the selected steels from SAE J1099 [8].

\begin{tabular}{|c|c|c|c|c|c|c|c|c|}
\hline Materials & $E(\mathrm{GPa})$ & $\sigma_{\mathrm{y}}(\mathrm{MPa})$ & $\sigma_{\mathrm{u}}(\mathrm{MPa})$ & $\sigma_{\mathrm{f}}(\mathrm{MPa})$ & RA (\%) & $\varepsilon_{\mathrm{f}}$ & $\mathrm{HB}$ & $\sigma_{\mathrm{L}}(\mathrm{MPa})$ \\
\hline 1008 & 207 & 234 & 331 & 587.5 & 77.5 & 1.49 & 86 & 92.7 \\
\hline 1008 & 203 & 255 & 365 & 649.3 & 77.9 & 1.51 & 90 & 100.3 \\
\hline 1010 & 203 & 200 & 331 & 597.1 & 80.4 & 1.63 & 83 & 116.9 \\
\hline 1015 & 207 & 228 & 414 & 695.5 & 68 & 1.14 & 80 & 146.3 \\
\hline 1020 & 203 & 262 & 441 & 713.5 & 61.8 & 0.96 & 109 & 143.9 \\
\hline 1020 & 186 & 255 & 393 & 644.5 & 64 & 1.02 & 108 & 129.5 \\
\hline 1025 & 207 & 306 & 547 & 889.4 & 62.6 & 0.98 & 158 & 197.8 \\
\hline 1045 & 207 & 517 & 752 & 1082.9 & 44 & 0.58 & 225 & 291.1 \\
\hline 1045 & 207 & 1689 & 1827 & 2758.8 & 51 & 0.71 & 500 & 690.3 \\
\hline 1045 & 207 & 1862 & 2241 & 3159.8 & 41 & 0.53 & 595 & 728.5 \\
\hline 1045 & 207 & 424 & 718 & 1062.6 & 48 & 0.65 & 192 & 227.9 \\
\hline 1045 & 207 & 1729 & 1956 & 2705.1 & 38.3 & 0.48 & 500 & 756.9 \\
\hline 1045 & 207 & 1275 & 1344 & 2137.0 & 59 & 0.89 & 390 & 512.6 \\
\hline 10B21 & 197 & 999 & 1048 & 1756.4 & 67.6 & 1.13 & 318 & 482.7 \\
\hline 10B21 & 203 & 806 & 834 & NA & NA & NA & 255 & 369.6 \\
\hline 10B22 & 203 & 806 & 834 & NA & NA & NA & 255 & 450.7 \\
\hline $15 \mathrm{~B} 27$ & 203 & 772 & 847 & 1431.4 & 69 & 1.17 & 250 & 410.2 \\
\hline $15 \mathrm{~B} 27$ & 203 & 854 & 916 & 1525.1 & 66.5 & 1.09 & 264 & 451.2 \\
\hline 4130 & 200 & 1358 & 1427 & 2207.6 & 54.7 & 0.79 & 366 & 549.4 \\
\hline 4130 & 221 & 778 & 896 & 1499.0 & 67.3 & 1.12 & 259 & 412.6 \\
\hline 4140 & 207 & 848 & 938 & NA & NA & NA & 293 & 473.1 \\
\hline 4140 & 200 & 1895 & 2033 & 2439.6 & 20 & 0.22 & 475 & 663.5 \\
\hline 4142 & 200 & 1447 & 1551 & 2280.0 & 47 & 0.63 & 400 & 528.2 \\
\hline 4142 & 200 & 1860 & 1929 & 2642.7 & 37 & 0.46 & 450 & 597.0 \\
\hline 4142 & 207 & 1378 & 1413 & 2091.2 & 48 & 0.65 & 380 & 547.9 \\
\hline 4142 & 207 & 1584 & 1757 & 2494.9 & 42 & 0.54 & 450 & 643.1 \\
\hline 4142 & 207 & 1722 & 1929 & 2604.2 & 35 & 0.43 & 475 & 667.2 \\
\hline 4340 & 200 & 1371 & 1468 & 2027.3 & 38.1 & 0.48 & 409 & 540.3 \\
\hline 4340 & 190 & 834 & 1048 & NA & NA & NA & 275 & 429.8 \\
\hline 4340 & 193 & 634 & 827 & 1185.9 & 43.4 & 0.57 & 243 & 301.9 \\
\hline 4340 & 207 & 1102 & 1171 & 1826.8 & 56 & 0.82 & 327 & 502.2 \\
\hline 5160 & 203 & 1488 & 1584 & 2212.8 & 39.7 & 0.51 & 430 & 634.2 \\
\hline 0030 & 207 & 303 & 496 & 724.2 & 46 & 0.62 & 137 & 196.5 \\
\hline 0050A & 209 & 415 & 787 & 936.5 & 19 & 0.21 & 192 & 211.9 \\
\hline 0050A & 209 & 402 & 583 & 734.6 & 26 & 0.30 & 174 & 200.7 \\
\hline $0050 \mathrm{~A}$ & 211 & 542 & 702 & NA & NA & NA & 206 & 258.0 \\
\hline 8630 & 207 & 985 & 1144 & 1475.8 & 29 & 0.34 & 305 & 334.6 \\
\hline
\end{tabular}




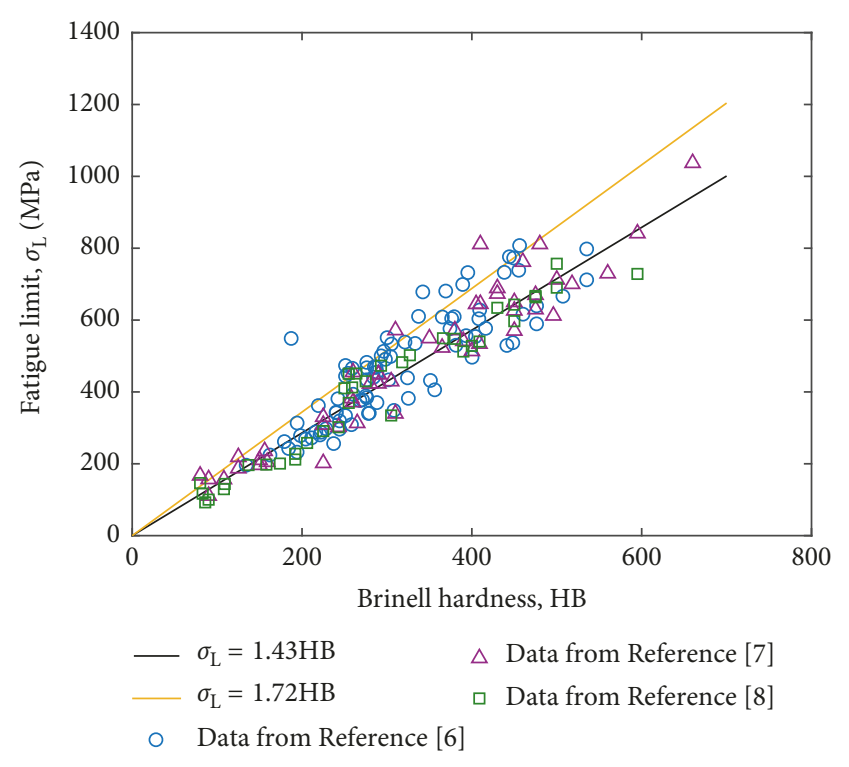

Figure 2: Fatigue limit vs. Brinell hardness.

If Basquin's equation is used to model the S-N curve, the fatigue limit can be determined by the following equation at $10^{6}$ cycles:

$$
\sigma_{\mathrm{L}}=\sigma_{\mathrm{f}}^{\prime}\left(2 \times 10^{6}\right)^{\mathrm{b}}
$$

In the absence of tested data, the fatigue strength coefficient, $\sigma_{\mathrm{f}}^{\prime}$, and the fatigue strength exponent, $b$, can be estimated using the monotonic tensile properties or Brinell hardness [4,9-12]. Then, the fatigue limit, $\sigma_{\mathrm{L}}$, can also be estimated indirectly by substituting the predicted $\sigma_{\mathrm{f}}^{\prime}$ and $b$ into equation (6). This method will be hereafter referred to as indirect method.

Based on the indirect method, the fatigue strength coefficient and the fatigue strength exponent can be predicted by six existing estimation methods [4,9-12] firstly, and then the fatigue limit is calculated using equation (6). The six existing estimation methods considered in this paper are listed in Table 4 . In this table, $E$ is the modulus of elasticity, $\sigma_{\mathrm{u}}$ is the ultimate tensile strength, $\mathrm{HB}$ is the Brinell hardness, $\sigma_{\mathrm{f}}$ is the true fracture strength, and $\varepsilon_{\mathrm{f}}$ is the true fracture ductility.

\section{Evaluation of the Estimation Methods}

As analyzed above, the direct methods proposed by Roessle and Fatemi [4] give better predictions than the methods shown in References [1, 3]. Therefore, the direct methods proposed by Roessle and Fatemi [4] are evaluated by using the tested data of 171 steels. The predicted fatigue limit, $\sigma_{\text {Pre, }}$ is compared with the experimental one, $\sigma_{\text {Exp }}$, in Figure 3 . For comparison purpose, the same tested data are also used to evaluate the aforementioned six indirect methods (Figure 4). In these figures, the dotted lines, solid lines, and dasheddotted lines represent factors of $\pm 10 \%, \pm 20 \%$, and $\pm 30 \%$ scatter band, respectively. It can be seen that, for most of considered steels, the MFPCM-based indirect method tends to overestimate the fatigue limit, while the FPCM-based and USM-based indirect methods as well as the $\sigma_{\mathrm{u}}$-based direct method tend to underestimate the fatigue limit. Both MUSM-based and HM-based indirect methods tend to overestimate the fatigue limit when the experimental fatigue limit is low (about the experimental fatigue limit less than $400 \mathrm{MPa}$ ), while underestimations are presented when the experimental fatigue limit is high (about the experimental fatigue limit larger than $400 \mathrm{MPa}$ ). As for the HB-based direct method, it may be said to provide the best estimation results among all the eight estimation methods.

Most of the work done in comparing both the direct and indirect methods use the scatter band criterion where the percentage of predicted fatigue limit versus experimental data falling within a scatter band is compared (Table 5). Factors of $\pm 10 \%, \pm 20 \%$, and $\pm 30 \%$ deviation from tested values are included in this table, along with the percentages of the data within each factor. It can be seen that the HBbased direct method has the most data with all deviation factors. For example, $86.5 \%$ of the predicted values are within a factor of $\pm 20 \%$ of the experimental values based on the HB-based direct method. In contrast, the best results predicted by MM-based method among the considered indirect methods from the literature are $76.6 \%$ of the predicted fatigue limit within the same factor of experimental values.

It should be noted here that one of the shortcomings of the scatter band criterion is that the trend of the correlation between prediction and experiment cannot be captured. Comparison of the HB-based and $\sigma_{\mathrm{u}}$-based direct methods with experimental data using scatter band criterion is schematically shown in Figure 5. It is observed that both the HBbased and $\sigma_{\mathrm{u}}$-based direct methods predict well within the scatter band and hence have to be considered as equal. However, the trend of the HB-based direct method with experimental data is closer than that of the $\sigma_{\mathrm{u}}$-based direct method, which is not captured by the scatter band criterion. To overcome this drawback, the standard deviation criterion is introduced to compare different estimation methods:

$$
S_{\mathrm{D}}=\sqrt{\frac{1}{n_{\mathrm{p}}-1} \sum_{i=1}^{n_{\mathrm{p}}}\left[\ln \left(\sigma_{\operatorname{Pr} e}\right)-\ln \left(\sigma_{\text {Exp }}\right)\right]^{2}},
$$

where $n_{\mathrm{p}}$ is the number of experimental data. It can be seen that the bigger the $S_{\mathrm{D}}$ is, the more scattered the predictions are. The comparisons using the standard deviation criterion are also tabulated in Table 5. It is shown that the HB-based direct method provides the best predictions.

In order to have a better quantitative comparison, the comparisons for different estimation methods are also given in the format of fitted probability density functions (PDF) for the predicted errors. For the statistical study, each data set is sorted in ascending order, and then each data point is associated with its mean rank. After that, each data set is fitted using the selected probability distribution. Here, the log-logistic distribution is selected based on the recommendation of Meggiolaro and Castro [12]. The cumulative distribution function of the log-logistic distribution function is given by the following equation [13] 
TABLE 4: Approximation methods for Basquin's fatigue properties.

\begin{tabular}{lcc}
\hline Approximation methods & $\sigma_{\mathrm{f}}^{\prime}(\mathrm{MPa})$ & $b$ \\
\hline Four-point correlation method (FPCM) [9] & $1.12 \sigma_{\mathrm{u}}\left(\sigma_{\mathrm{f}} / \sigma_{\mathrm{u}}\right)^{0.893}$ & $-0.0792-0.179 \lg \left(\sigma_{\mathrm{f}} / \sigma_{\mathrm{u}}\right)$ \\
Universal slopes method (USM) [9] & $1.9018 \sigma_{\mathrm{u}}$ & -0.12 \\
Modified universal slopes method (MUSM) [10] & $E \times 0.623\left(\sigma_{\mathrm{u}} / E\right)^{0.832}$ & -0.09 \\
Modified four-point correlation method (MFPCM) & $\sigma_{\mathrm{u}}\left(1+\varepsilon_{\mathrm{f}}\right)$ & $\frac{1}{6}\left\{\lg \left[0.16\left(\sigma_{\mathrm{u}} / E\right)^{0.81}\right]-\lg \left(\sigma_{\mathrm{f}} / E\right)\right\}$ \\
{$[11]$} & $1.5 \sigma_{\mathrm{u}}$ & -0.09 \\
Medians method (MM) [12] & $4.25 \mathrm{HB}+225$ & -0.09 \\
Hardness method (HM) [4] & & \\
\hline
\end{tabular}

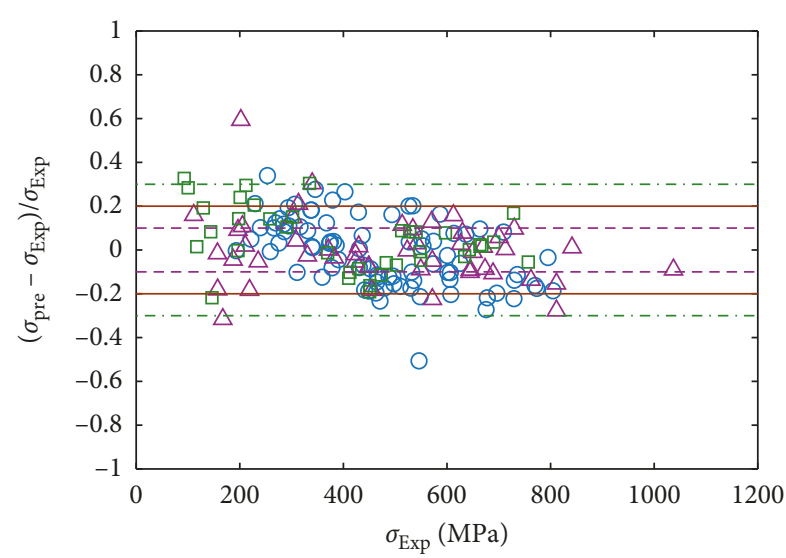

○ Data from Reference [6]

$\triangle$ Data from Reference [7]

$\square$ Data from Reference [8]

(a)

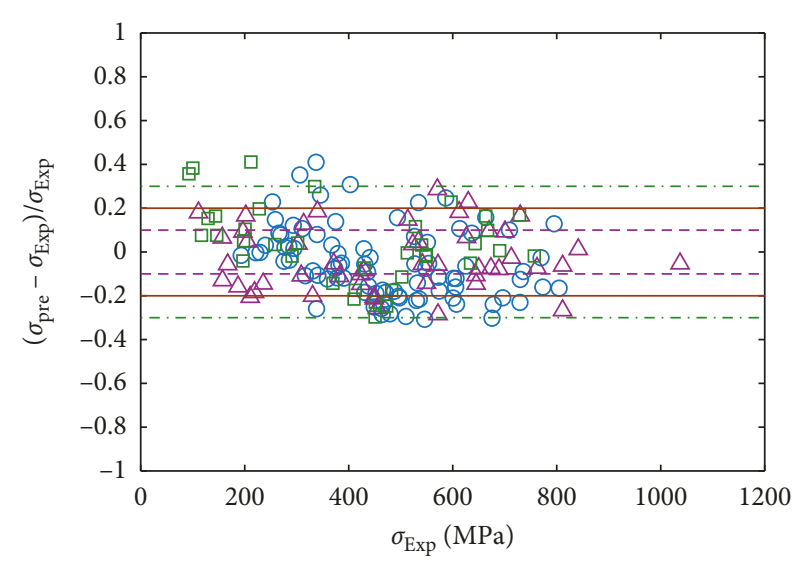

○ Data from Reference [6]

$\triangle$ Data from Reference [7]

口 Data from Reference [8]

(b)

Figure 3: Comparison of the direct methods using the tested data from [6-8]: (a) HB-based and (b) $\sigma_{\mathrm{u}}$-based.

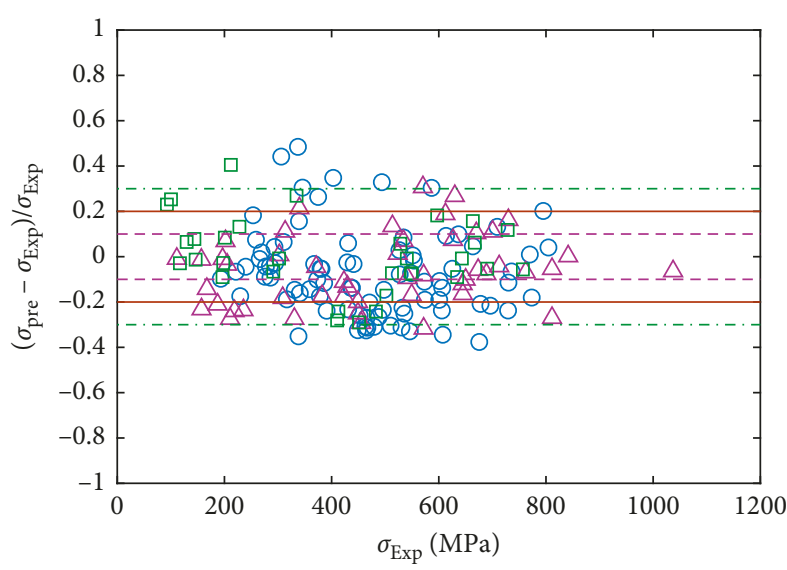

- Data from Reference [6]

$\triangle$ Data from Reference [7]

$\square$ Data from Reference [8]

(a)

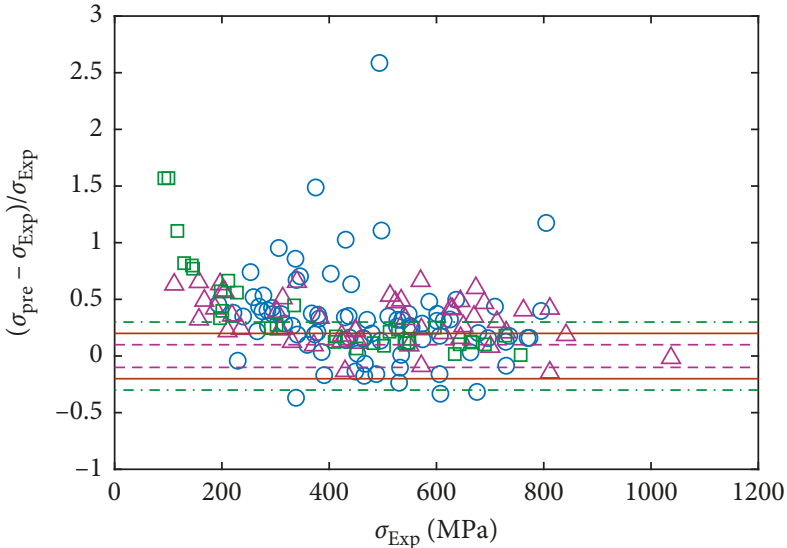

$\triangle$ Data from Reference [6]
$\triangle$ Data from Reference [7]
$\square$ Data from Reference [8]

(b)

FIgURE 4: Continued. 


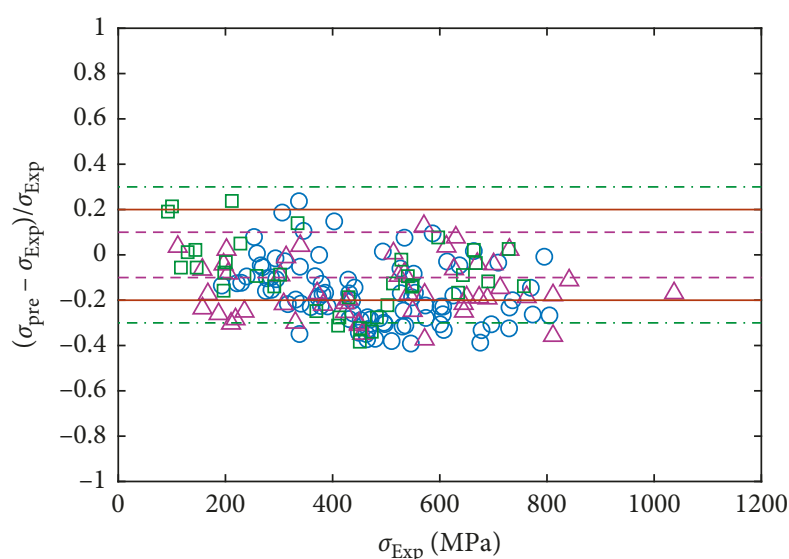

○ Data from Reference [6]

$\triangle$ Data from Reference [7]

$\square$ Data from Reference [8]

(c)

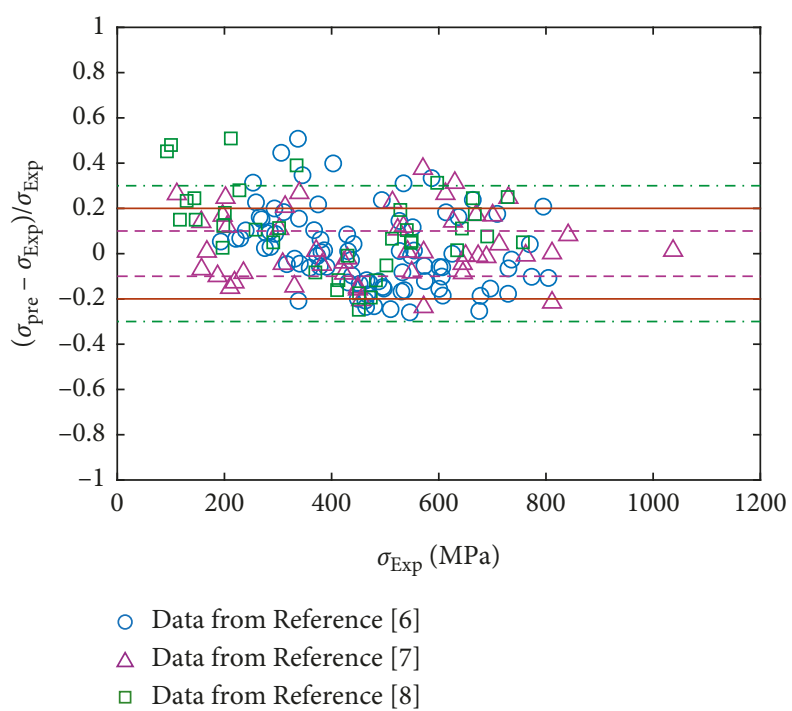

(e)

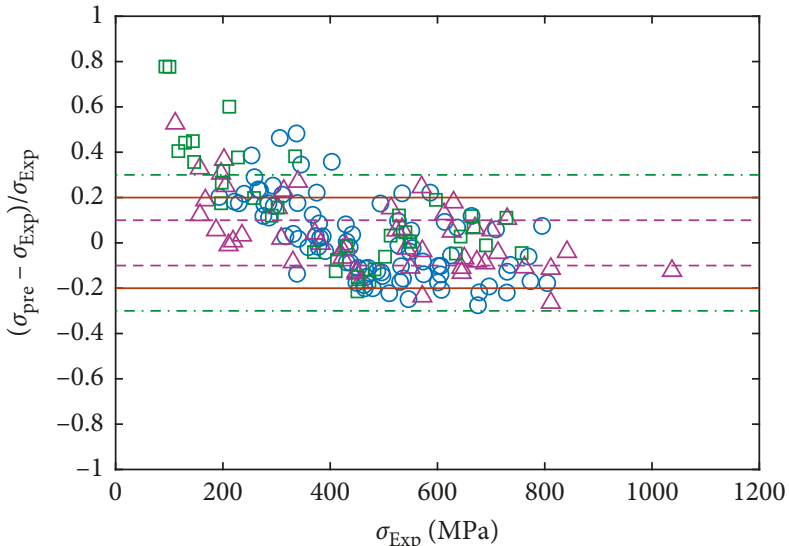

- Data from Reference [6]

$\triangle$ Data from Reference [7]

$\square$ Data from Reference [8]

(d)

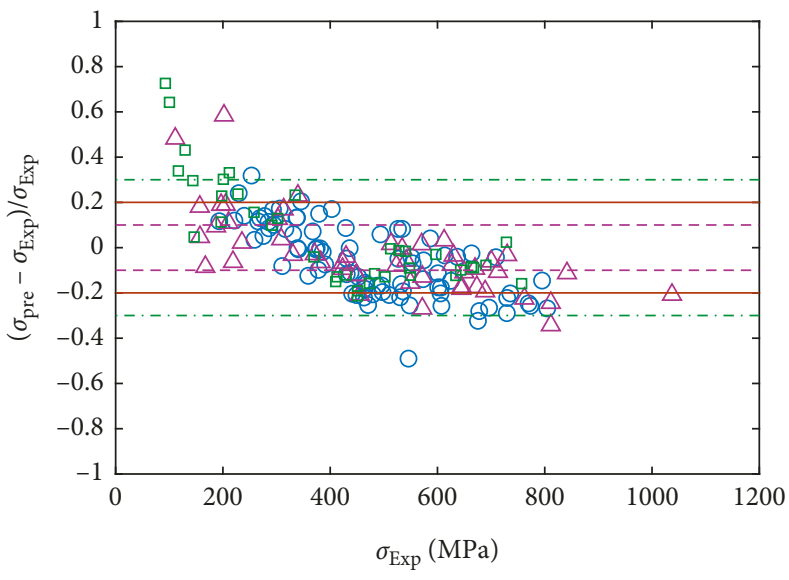

○ Data from Reference [6]

$\triangle$ Data from Reference [7]

$\square$ Data from Reference [8]

(f)

Figure 4: Comparison of the indirect methods using the tested data from [6-8]: (a) FPCM-based method, (b) MFPCM-based method, (c) USM-based method, (d) MUSM-based method, (e) MM-based method, and (f) HM-based method.

TABLE 5: Comparison of estimation methods in terms of evaluation values.

\begin{tabular}{lcccccccc}
\hline & HB-based & $\sigma_{\mathrm{u}}$-based & FPCM & MFPCM & USM & MUSM & MM & HM \\
\hline $\pm 10 \%$ & 0.544 & 0.450 & 0.440 & 0.102 & 0.327 & 0.392 & 0.427 & 0.392 \\
$\pm 20 \%$ & 0.865 & 0.772 & 0.687 & 0.361 & 0.608 & 0.754 & 0.766 & 0.766 \\
$\pm 30 \%$ & 0.977 & 0.965 & 0.892 & 0.512 & 0.877 & 0.889 & 0.918 & 0.895 \\
$S_{\mathrm{D}}$ & 0.155 & 0.170 & 0.199 & 0.353 & 0.240 & 0.182 & 0.167 & 0.187 \\
\hline
\end{tabular}

$$
F(x)=\frac{x^{p}}{a^{p}+x^{p}}, \quad x>0, a>0, p \geq 1
$$

where $p$ is a shape parameter and $a$ is a scale parameter. The corresponding probability density function (PDF) of the loglogistic distribution is given by [13]

$$
f(x)=\frac{p a^{p} x^{p-1}}{\left(a^{p}+x^{p}\right)^{2}}, \quad x>0, a>0, \quad p \geq 1 .
$$

The cumulative probability $P_{\mathrm{f}}$ of estimated data is evaluated by the mean rank method as

$$
P_{\mathrm{f}}=\frac{i}{I+1}
$$

where $i$ is the rank number and $I$ is the total number of considered steels. Symbols show the $\sigma_{\text {Pre }} / \sigma_{\operatorname{Exp}}$ ratio of the considered eight estimation methods (Figure 6). Optimum values of the parameters $a$ and $p$ are obtained by applying 


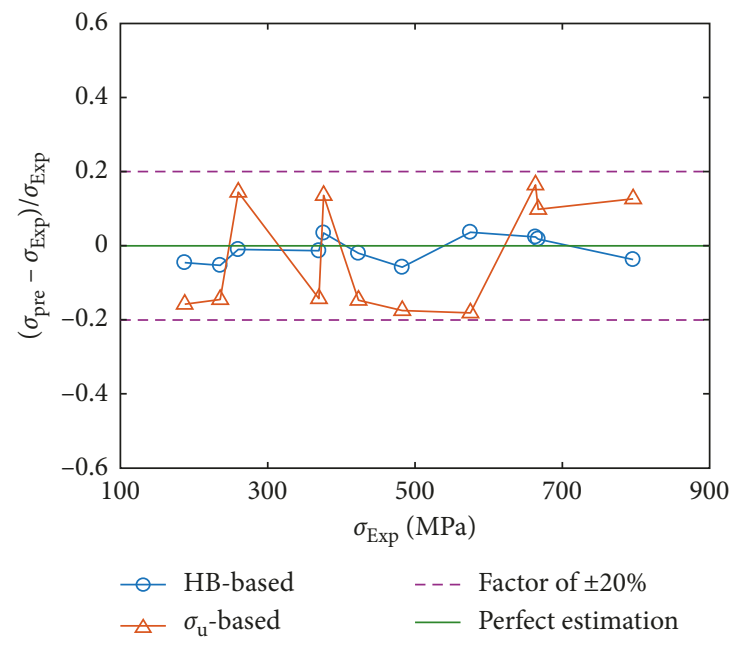

FIGURE 5: Schematic showing the limitation in comparing two different methods using scatter band criterion.

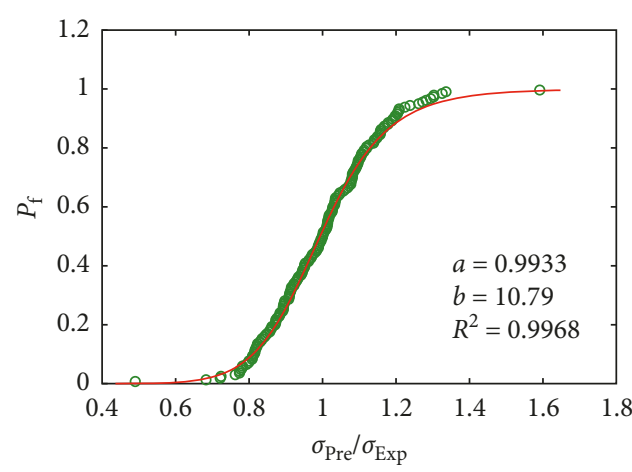

(a)

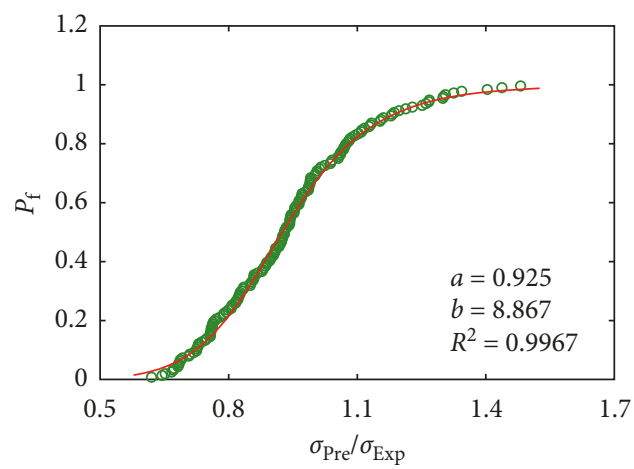

(c)

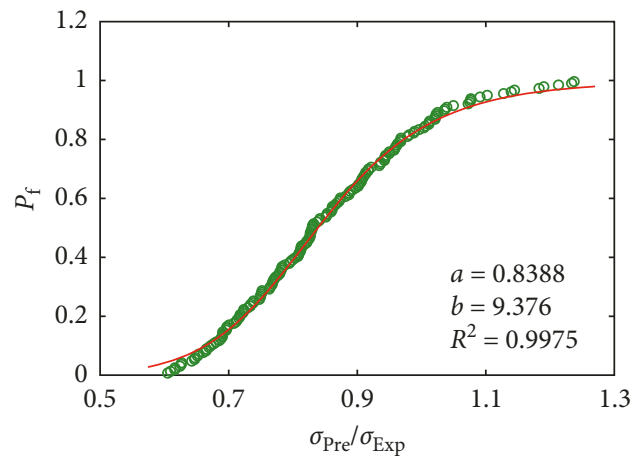

(e)

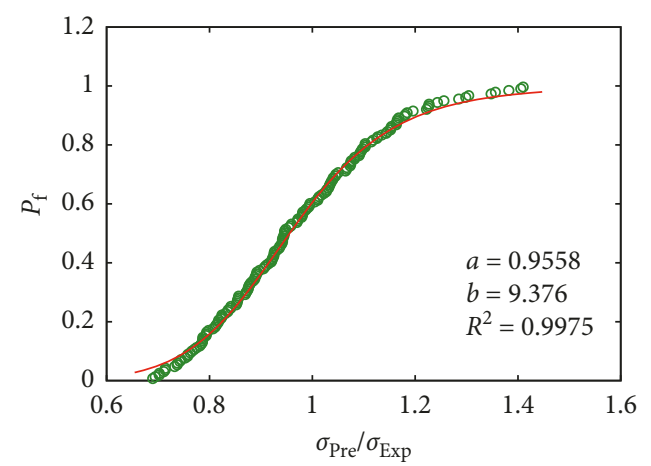

(b)

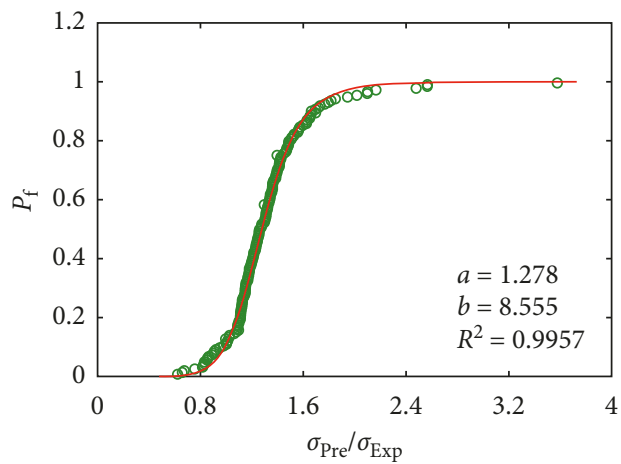

(d)

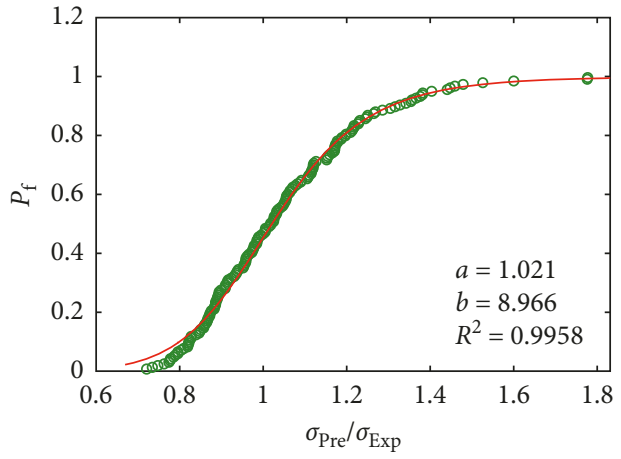

(f)

Figure 6: Continued. 


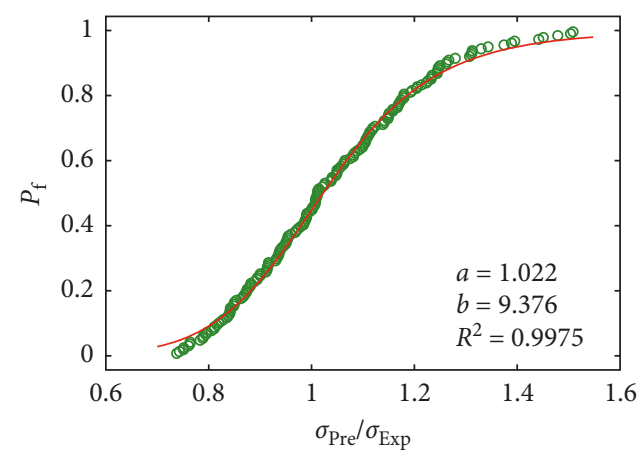

(g)

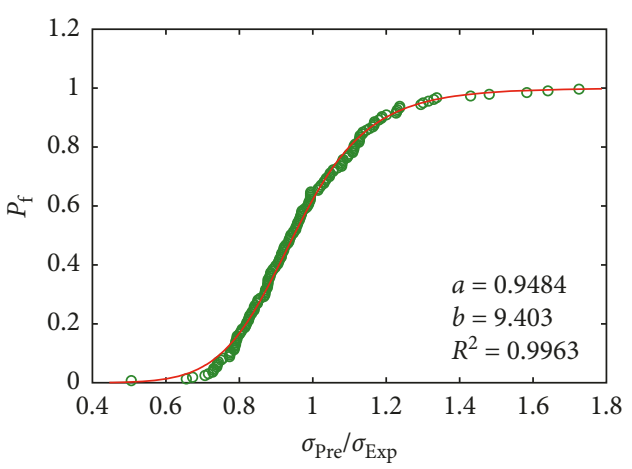

(h)

Figure 6: The estimates of the considered estimation methods (the curvilinear lines show the fit of log-logistic distribution): (a) HB-based method, (b) $\sigma_{\mathrm{u}}$-based method, (c) FPCM-based method, (d) MFPCM-based method, (e) USM-based method, (f) MUSM-based method, (g) MM-based method, and (h) HM-based method.

the least squares method [14]. The curvilinear lines indicate the results of fitting (Figure 6). The optimum values of $a$ and $p$ are also presented in Figure 6. Then, the best-fitted distributions of different estimation methods are shown in Figure 7. It is clearly shown that the FPCM-based and USM-based indirect methods as well as the $\sigma_{\mathrm{u}}$-based direct method provide underestimations, while the MFPCMbased indirect method provides overestimation. The HBbased direct method provides reasonable predictions while somewhat underestimations are shown in Figure 7. In contrast to the considered estimation methods, the HBbased direct method on the basis of Brinell hardness provides the best predictions for the fatigue limits of most of steels.

Although the HB-based direct method presents the best predictions among the considered estimation methods, it is known that, for advanced steels, the predictability of the fatigue limit based on monotonic properties often fails. The failed predictions may lie in the high notch sensitivity since a lot of the advanced steels belong to the high-strength materials. The inclusions act as micronotches in the material which can generate fatigue crack nuclei, especially in highstrength material [15]. Because of the high-yield strength of these materials, they have a high notch sensitivity, even for micronotches [15]. Therefore, in high-strength steel, inclusions are more harmful because of the higher notch sensitivity. Then, the predictability of the fatigue limit based on monotonic properties for advanced steels is more challengeable, which is needed to be studied further.

\section{Conclusions}

(1) The Brinell hardness, HB, can correlate the fatigue limit well. The fatigue limit can be estimated well by the HB-based direct method for most of considered steels. In the absence of $\mathrm{HB}$, the $\sigma_{\mathrm{u}}$-based direct method is recommended to calculate the fatigue limit.

(2) In contrast to the direct methods, all the indirect methods are not only relatively complicated to use but also more scattered in predictions.

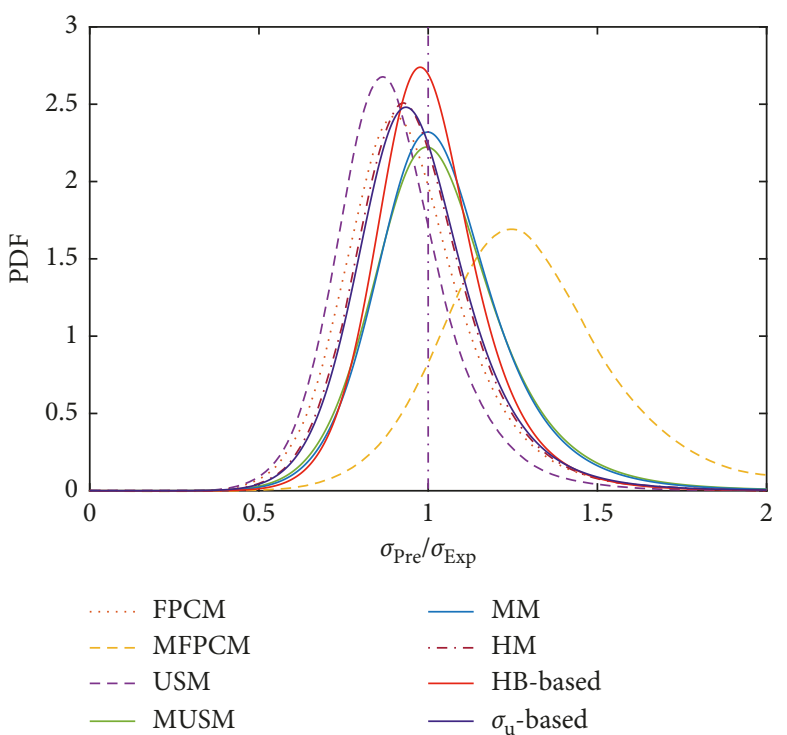

Figure 7: Probability density functions of the considered estimation methods.

Finally, it is important to point out that HB-based direct method is not physical law although it correlates the fatigue limit of many steels well. It is still an empirical equation. Therefore, such estimates should not be used to replace experiments. Whenever possible, the fatigue limit should be obtained from the tested data. These estimates, even the HBbased direct method, are only admissible during the first stage of design.

\section{Data Availability}

The data used to support the findings of this study are included within the article.

\section{Conflicts of Interest}

The authors declare that they have no conflicts of interest. 


\section{Acknowledgments}

The authors gratefully acknowledge the financial support of the National Natural Science Foundation of China (No. 51601221), the Natural Science Basic Research Plan in Shaanxi Province of China (No. 2019JQ-353), the Fundamental Research Funds for the Central Universities (No. JB180402), and the National Defense Basic Scientific Research Project (JSZL2017203C012).

\section{References}

[1] R. I. Stephens, A. Fatemi, R. R. Stephens, and H. O. Fuchs, Metal Fatigue in Engineering, John Wiley \& Sons, Hoboken, NJ, USA, 2nd edition, 2000.

[2] F. C. Campbell, Fatigue and Fracture: Understanding the Basics, ASM International, Novelty, OH, USA, 2012.

[3] D. F. Socie, M. R. Mitchell, and E. M. Caulfield, "Fundamentals of modern fatigue analysis," Fracture Control Program Report No. 26, University of Illinois, Champaign, IL, USA, 1977.

[4] M. Roessle and A. Fatemi, "Strain-controlled fatigue properties of steels and some simple approximations," International Journal of Fatigue, vol. 22, no. 6, pp. 495-511, 2000.

[5] Z. Lopez, "Correlations among tensile and cyclic deformation properties for steels and implications on fatigue life predictions," M.S. thesis, The University of Toledo, Toledo, OH, USA, 2012.

[6] American Iron and Steel Institute, Bar Steel Fatigue Database, American Iron and Steel Institute, Washington, DC, USA, 2018, http://barsteelfatigue.autosteel.org/.

[7] American Society for Metals, ASM Handbook Volume 1-Properties and Selection: Irons and steels, ASM International, Novelty, OH, USA, 9th edition, 1978.

[8] Society of Automotive Engineers, "Technical report on low cycle fatigue properties: ferrous and non-ferrous materials," SAE J1099, Society of Automotive Engineers, Pennsylvania, MI, USA, 2002.

[9] S. S. Manson, "Fatigue: a complex subject-some simple approximation," Experimental Mechanics, vol. 5, no. 4, pp. 193-226, 1965.

[10] U. Muralidharan and S. S. Manson, "A modified universal slopes equation for estimation of fatigue characteristics of metals," Journal of Engineering Materials and Technology, vol. 110, no. 1, pp. 55-58, 1988.

[11] J. Ong, "An improved technique for the prediction of axial fatique life from tensile data," International Journal of Fatigue, vol. 15, no. 3, pp. 213-219, 1993.

[12] M. Meggiolaro and J. T. P. Castro, "Statistical evaluation of strain-life fatigue crack initiation predictions," International Journal of Fatigue, vol. 26, no. 5, pp. 463-476, 2004.

[13] F. Ashkar and S. Mahdi, "Fitting the log-logistic distribution by generalized moments," Journal of Hydrology, vol. 328, no. 3-4, pp. 694-703, 2006.

[14] V. L. Huy, J. Gaspar, O. Paul, and S. Kamiya, "Statistical characterization of fatigue lifetime of polysilicon thin films," Sensors and Actuators A: Physical, vol. 179, pp. 251-262, 2012.

[15] J. Schijve, Fatigue of Structures and Materials, Springer, Berlin, Germany, 2nd edition, 2009. 


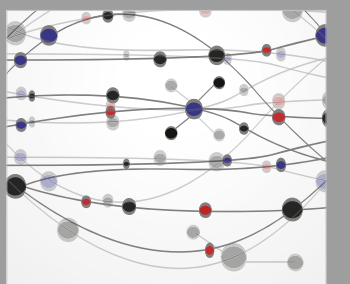

The Scientific World Journal
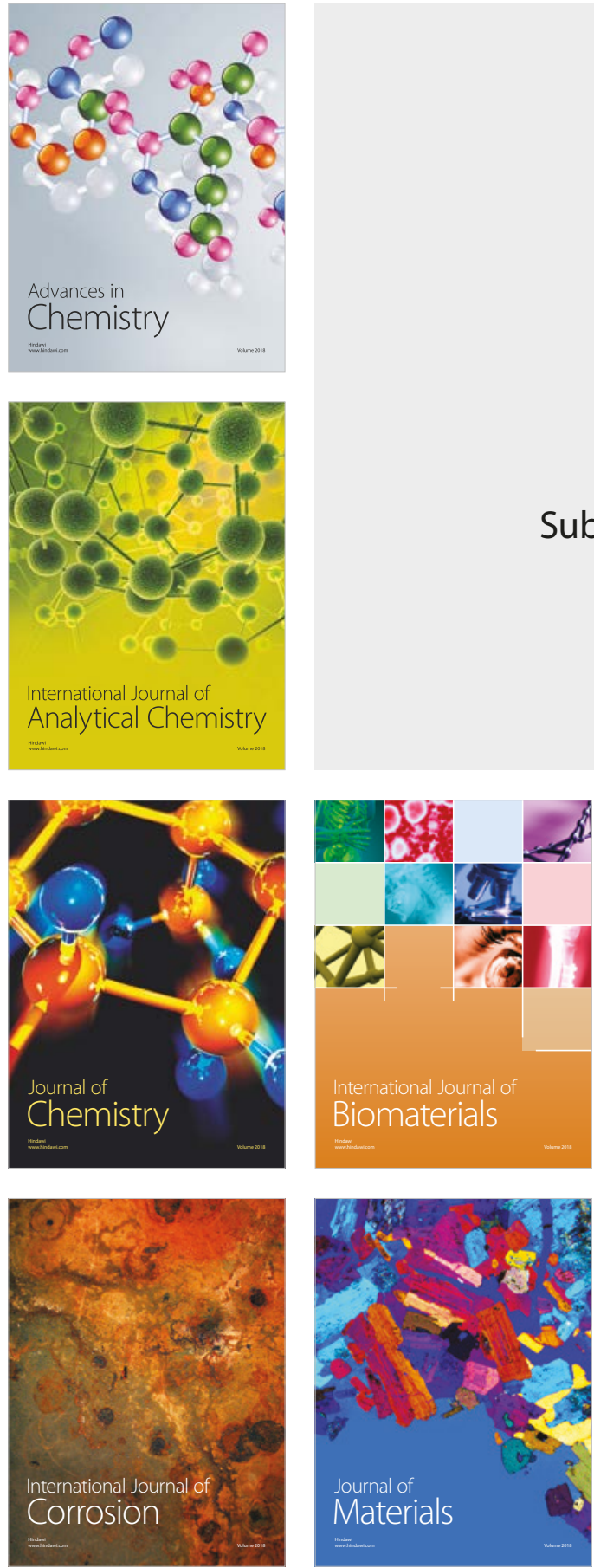

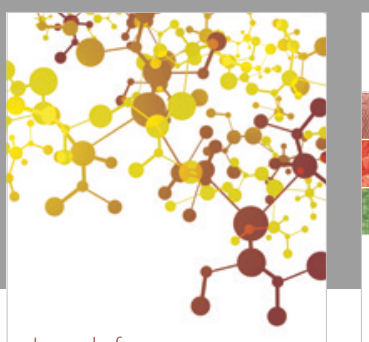

Journal of

Applied Chemistry
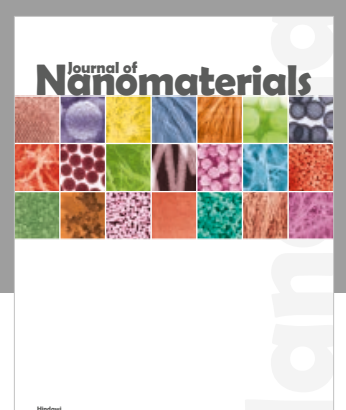

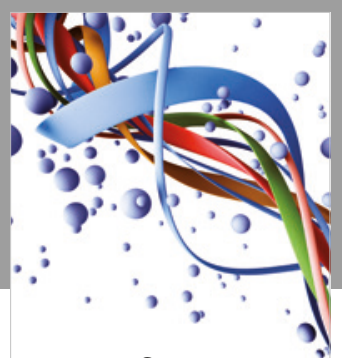

Scientifica

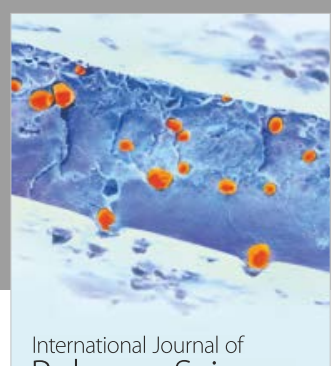

Polymer Science

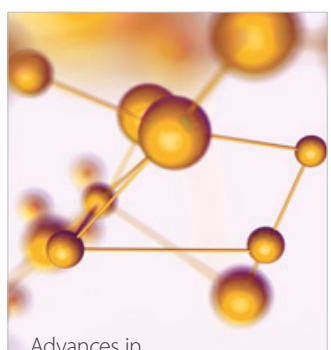

Physical Chemistry
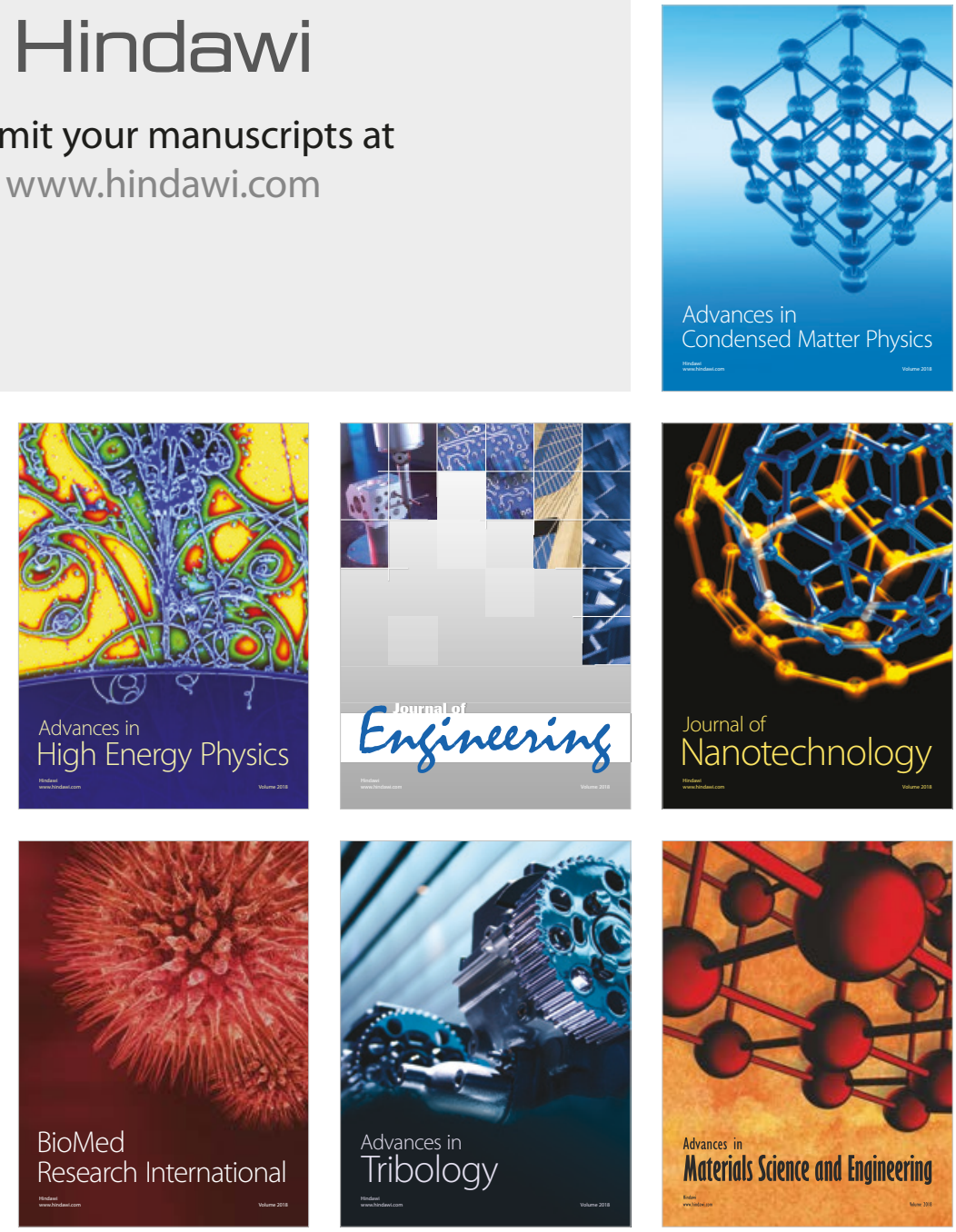